LAPP-EXP 2002-08

\title{
Fermion pair production at LEP2
}

\author{
I. DE BONIS \\ LAPP, IN2P3-CNRS, Chemin de Bellevue, BP110, \\ F-74941, Annecy-le-Vieux
}

\begin{abstract}
Preliminary results on combined cross-sections and forward-backward asymmetries from $e^{+} e^{-} \rightarrow f \bar{f}$ processes at LEP2 for center-of-mass energy $\sqrt{s}$ from 130 to $207 \mathrm{GeV}$, are summarized. These results provide a test of the Standard Model to $\mathcal{O}(1 \%)$ and allow to constraint physics beyond the Standard Model, such as four-fermion contact interactions, gravitational interaction in extra dimensions and leptoquarks models.
\end{abstract}

Talk given at the $31^{\text {th }}$ International Conference for High Energy Physics, 24-31 july 2002, Amsterdam, The Netherlands 


\section{Fermion pair production at LEP2}

\section{De Bonis ${ }^{\mathrm{a}}$}

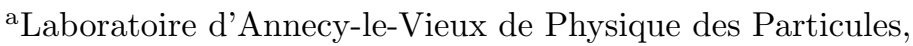
74941 Annecy-Le-Vieux Cedex, France

Preliminary results on combined cross-sections and forward-backward asymmetries from $e^{+} e^{-} \rightarrow f \bar{f}$ processes at LEP2 for center-of-mass energy $\sqrt{s}$ from 130 to $207 \mathrm{GeV}$, are summarized. These results provide a test of the Standard Model to $\mathcal{O}(1 \%)$ and allow to constraint physics beyond the Standard Model, such as four-fermion contact interactions, gravitational interaction in extra dimensions and leptoquarks models.

\section{INTRODUCTION}

Since the start of LEP2, each experiment has collected data for a total integrated luminosity of about $700 p b^{-1}$, at center-of-mass energy $\sqrt{s}$ from 130 to $207 \mathrm{GeV}$ [1]. In this paper, combined difermion results from the four LEP experiments up to $207 \mathrm{GeV}$ are presented. Then, constraints on physics beyond the Standard Model such as fourfermion contact interactions, gravitational interaction in extra dimensions and leptoquarks are reported.

\section{FERMION PAIR PRODUCTION AT LEP2}

The process $e^{+} e^{-} \rightarrow f \bar{f}$ at energies above the $Z^{0}$ resonance is dominated by the $\gamma$ exchange. On the other hand the $\gamma-Z^{0}$ interference is responsible for the large forward-backward asymmetries in all channels. At LEP2 energies, compared to LEP1, the importance of radiative processes in which an initial state photon is emitted, is much increased. This initial-state radiation (ISR) reduces the available center-of-mass energy $\sqrt{s^{\prime}}$ down to $Z^{0}$ resonance and QED radiative corrections lead to a factor five enhancement of the di-fermion Born cross-section. The reduced center-of-mass energy $\sqrt{s^{\prime}}$ is defined as the invariant mass of the outgoing $l \bar{l}$ pair or the mass of the s-channel propagator for $q \bar{q}$ pair.

The di-fermion measurements presented below are provided for the exclusive samples of events with $\sqrt{s^{\prime} / s}>0.85$, so excluding the radiative events.

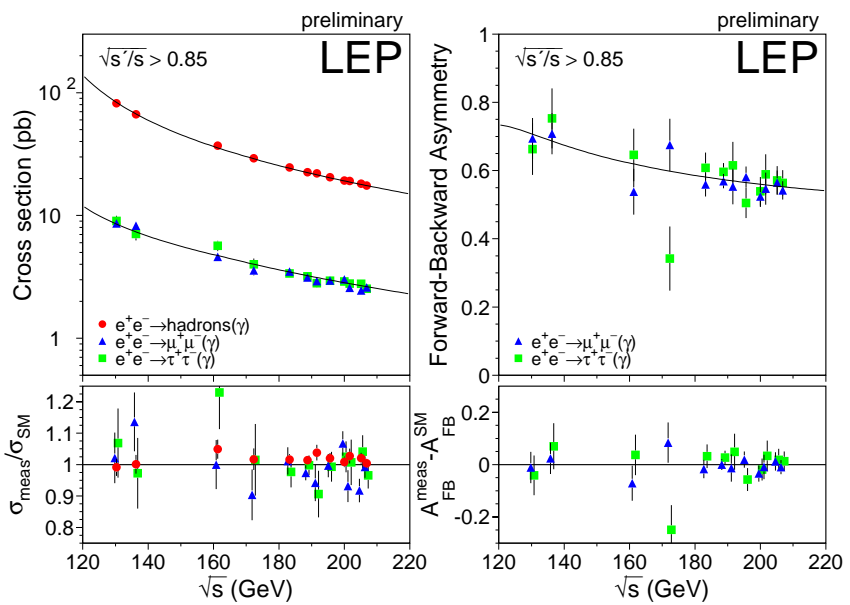

Figure 1. Preliminary LEP combined crosssections and asymmetries results

\section{DI-FERMION CROSS-SECTIONS AND LEPTONIC ASYMMETRIES WITH $\sqrt{s^{\prime} / s}>0.85$}

Each LEP experiment provided its measurements of the $q \bar{q}, \mu^{+} \mu^{-}$and $\tau^{+} \tau^{-}$cross-sections and of $\mu^{+} \mu^{-}$and $\tau^{+} \tau^{-}$asymmetries. Before combination, those measurements are corrected 
to have a common signal definition. The combination includes data from 183 to $207 \mathrm{GeV}$. All cross-sections and asymmetries are treated together in a single fit using a covariance matrix (detectors effects and ISR modeling being the main errors). The LEP preliminary combined results are shown in Figure 1. The difference between the measured hadronic cross section and the Standard Model expectation (ZFITTER v6.36 [2]), averaged over all energies, is the largest one and is about 1.6 standard deviation. Theoretical precisions are respectively $0.3 \%$ and $0.4 \%$ for the hadronic and leptonic cross sections. They are obtained from the difference between ZFITTER and KK2f [3] predictions.

Differential cross-sections $d \sigma / d \cos \theta$ for $e^{+} e^{-} \rightarrow l \bar{l}$ processes are measured by the four LEP experiments. For $\mu^{+} \mu^{-}$and $\tau^{+} \tau^{-}$final states, preliminary results combination gives a good agreement with the Standard Model expectation (ZFITTER). A preliminary combination (ALEPH-L3OPAL) of the $e^{+} e^{-}$final states results have been made for the first time, averaged data and predictions from Monte Carlo generator BHWIDE [4] are in good agreement.

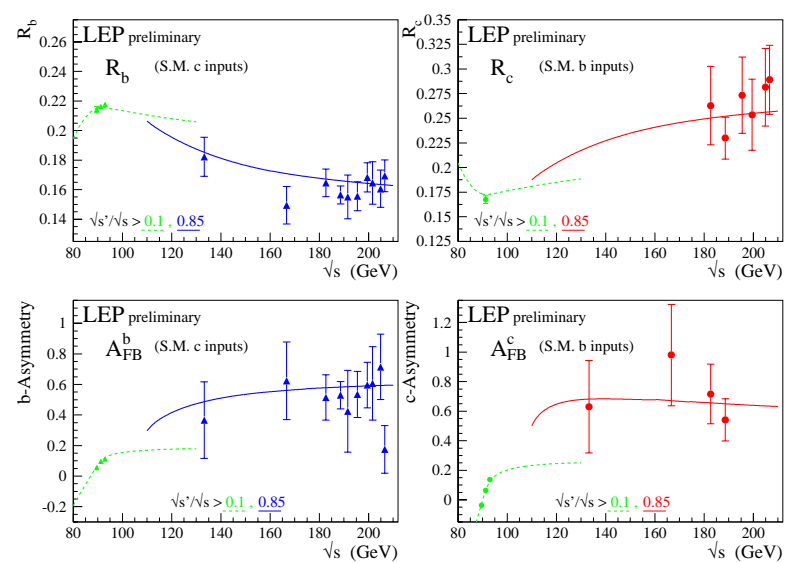

Figure 2. Preliminary LEP combined $R_{q}$ and $A_{F B}^{q \bar{q}}$ results for $b \bar{b}$ and $c \bar{c}$ production

\section{HEAVY FLAVOURS CROSS- SECTIONS AND ASYMMETRIES}

The measured observables are the cross section ratios $R_{b}=\sigma(b \bar{b}) / \sigma(\mathrm{had})$ and $R_{c}=$ $\sigma(c \bar{c}) / \sigma($ had), where $\sigma($ had $)$ is the total $q \bar{q}$ cross section, and the forward-backward asymmetries $A_{F B}^{b \bar{b}}$ and $A_{F B}^{c \bar{c}}$ (Figure 2). The available heavy flavours results are combined, $R_{c}$ measurements coming only from ALEPH experiment. A simultaneous fit of $R_{b}$ and $A_{F B}^{b \bar{b}}$ is performed, $R_{c}$ and $A_{F B}^{c \bar{c}}$ being fixed to the Standard model values. A similar procedure is used for the $c \bar{c}$ heavy flavour measurements. The results are consistent with the Standard Model predictions of ZFITTER.

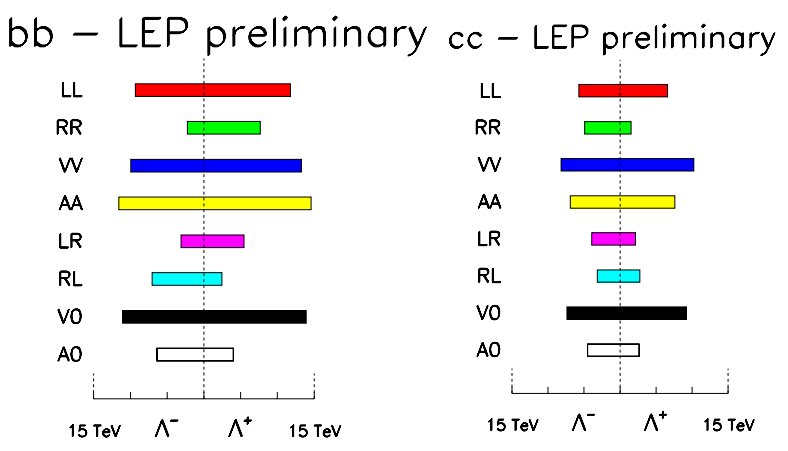

Figure 3. Preliminary combined $95 \%$ confidence limits on $\Lambda$ for $b \bar{b}$ and $c \bar{c}$. The \pm sign on $\Lambda$ denotes positive and negative interference with Standard Model amplitudes.

\section{INTERPRETATIONS}

Comparaison of the measured di-fermion crosssections and asymmetries with Standard Model predictions allows to put limits on many possible extensions of the Standard Model. The sensitivity to these new models comes from their interference with the Standard Model processes through virtual exchange of new particles. 


\subsection{Four-fermions contact interactions}

Four-fermions contact interactions are characterized by an energy scale $\Lambda$, and a coupling $g . \Lambda$ is interpreted as the mass of a new heavy particle exchanged between the incoming and the outgoing fermion pairs. Different models correspond to the different helicity states involved in the interaction. LEP2 combined di-fermion measurements allow to constrain the scale $\Lambda$ for $e^{+} e^{-} \rightarrow l \bar{l}, b \bar{b}, c \bar{c}$ final states only reachable at LEP. Contact interactions would affect the total cross section and the asymmetries for $l \bar{l}\left(\mu^{+} \mu^{-}, \tau^{+} \tau^{-}\right)$and heavy flavours final states. Preliminary combined $95 \%$ confidence lower limits on $\Lambda$ are derived, assuming a coupling $g^{2}=4 \pi$ (Figure 3 ). For the first time at LEP2, $u \bar{u}$ and $d \bar{d}$ final states are studied using the hadronic cross section and assuming that only one quark flavour of up-type or downtype is affected by the contact interactions (Figure 4).

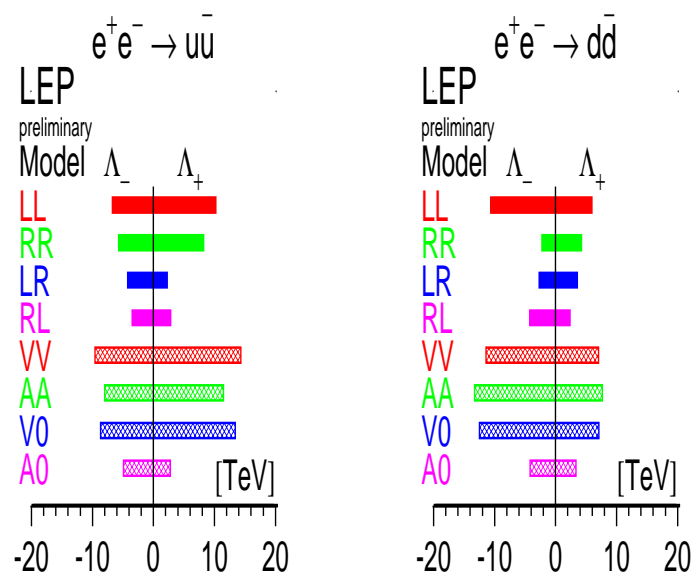

Figure 4. Preliminary combined 95\% confidence limits on $\Lambda$ for up-type and down-type quarks

\subsection{Gravitational interaction in extra di- mensions}

The gravitational interaction in extra dimensions are a new approach to understand the Hierarchy problem. These models, in particular the "Arkani-Ahmed, Dimopoulos, Dvali" model [5] assume $\delta$ extra space dimensions compactified at the size $\mathrm{R}$ and a fundamental scale $M_{D}$ close to the electroweak scale. Results are for $\delta=2$ and in term of string mass scale $M_{S} \sim M_{D}$ [6]. The $e^{+} e^{-} \rightarrow f \bar{f}$ Standard Model differential cross section is then modified due to additional s-channel virtual graviton exchange amplitudes. Especially the $e^{+} e^{-}$final state where the interference between t-channel $\gamma$ exchange and graviton exchange is expected to be large. The combined (ALEPH-L3-OPAL) preliminary 95\% confidence lower limits on $M_{S}$ from the $e^{+} e^{-} \rightarrow e^{+} e^{-}$measurements are the following:

$$
\begin{array}{ll}
M_{S}=1.20 \mathrm{TeV} & \text { for } \lambda=+1 \\
M_{S}=1.09 \mathrm{TeV} & \text { for } \lambda=-1
\end{array}
$$

where $\lambda$ is the sign of the interference with the SM amplitudes. The Figure 3 shows the measured $e^{+} e^{-} \rightarrow e^{+} e^{-}$differential cross-section normalized to the Standard Model expectation (Figure 5).

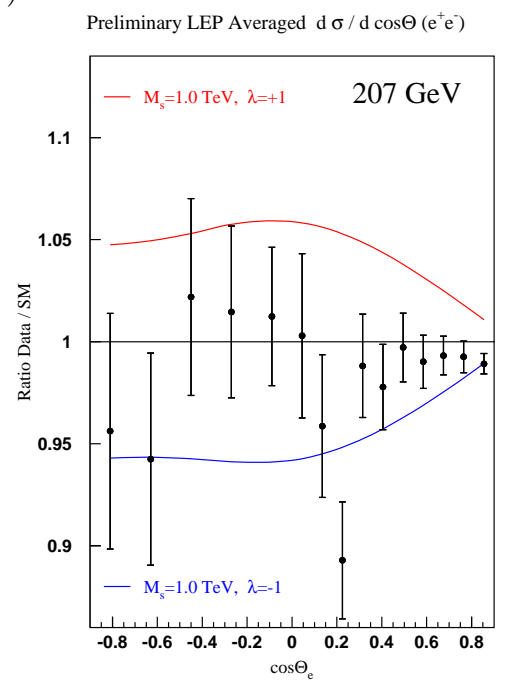

Figure 5. Measured $e^{+} e^{-} \rightarrow e^{+} e^{-}$differential cross-section normalized to the Standard Model expectation at $\sqrt{s}=207 \mathrm{GeV}$. The predictions for graviton interactions with $M_{S}=1 \mathrm{GeV}$ are indicated.

\subsection{Leptoquarks}

A leptoquark (LQ) is a high-mass resonant state carrying baryon and lepton numbers $(\mathrm{F}=3 \mathrm{~B}+\mathrm{L}$, where $\mathrm{F}$ is the LQ's fermion number) 
Table 1

Preliminary indirect limits on the first generation leptoquark mass, assuming $\lambda=\sqrt{4 \pi \alpha}$.

\begin{tabular}{llll}
\hline LQ Type & $m_{L Q}\left(G e V / c^{2}\right)$ & LQ Type & $m_{L Q}\left(G e V / c^{2}\right)$ \\
\hline$S_{0}(L) \rightarrow e u$ & 655 & $V_{1 / 2}(L) \rightarrow e d$ & 303 \\
$S_{0}(R) \rightarrow e u$ & 520 & $V_{1 / 2}(R) \rightarrow e u, e d$ & 227 \\
$\tilde{S}_{0}(R) \rightarrow e d$ & $\tilde{V}_{1 / 2}(L) \rightarrow e u$ & 176 \\
$S_{1}(L) \rightarrow e u, e d$ & $V_{0}(L) \rightarrow e \bar{d}$ & 917 \\
$S_{1 / 2}(L) \rightarrow e \bar{u}$ & 361 & $V_{0}(R) \rightarrow e \bar{d}$ & 165 \\
$S_{1 / 2}(R) \rightarrow e \bar{u}, e \bar{d}$ & 178 & $\tilde{V}_{0}(R) \rightarrow e \bar{u}$ & 489 \\
$\tilde{S}_{1 / 2}(L) \rightarrow e \bar{d}$ & 232 & $V_{1}(L) \rightarrow e \bar{u}, e \bar{d}$ & 659
\end{tabular}

and coupling to a lepton and a quark. They are predicted by theories such as the one proposed by W. Buchmüller, R. Rückl and D. Wyler [7]. Ten leptoquarks are predicted, five scalars $\left(S_{0}, S_{1 / 2}, \ldots\right)$, five vectors $\left(V_{0}, V_{1 / 2}, \ldots\right)$. There are three generations of LQ couplings, $\lambda_{l} q$. The
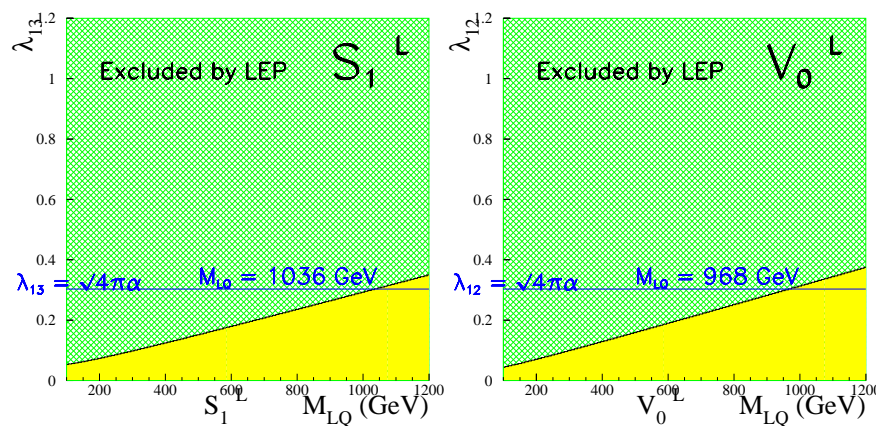

Figure 6. Preliminary limits in the plan $\lambda_{l q}^{2}$ versus LQ mass for $V_{0}(L)$ ( $2^{\text {nd }}$ generation coupling) and $S_{1}(L)$ ( $3^{r d}$ generation coupling)

$e^{+} e^{-} \rightarrow q \bar{q}$, leptoquarks can be exchanged in the t-channel or u-channel. The hadronic crosssection is modified by the first generation leptoquark exchange. $R_{c}$ and $A_{F B}^{c \bar{c}}$ measurements are modified by the second generation leptoquark exchange. $R_{b}$ and $A_{F B}^{b \bar{b}}$ measurements are sensitive to the third generation leptoquarks exchange. Assuming $\lambda=\sqrt{4 \pi \alpha}$, the indirect limits on the first generation leptoquark mass, are given in Table 1. For the second and third generation leptoquark exchange, Figure 6 shows the best LEP2 limits in the plan $\lambda_{l q}^{2}$ versus LQ mass.

\section{ACKNOWLEDGMENTS}

I would like to thank the members of the LEP2 Di-Fermion Electroweak Working Group.

\section{REFERENCES}

1. ALEPH collab. EPS-HEP2002 388, ALEPH CONF 2002-021, DELPHI 2001-168 PHYS 904, L3 collab. EPS-HEP2002, L3 Note 2763, OPAL TN704, LEPEWWG $f \bar{f}$ Subgroup, C. Geweniger et al., LEP2FF/01/02

2. D. Bardin et al, CERN-TH 6443/92; http://www.ifh.de/ riemann/Zfitter/zf.html.

3. S.Jadach, B.F. Ward and Z. Was, "The precision Monte Carlo event generator KK for two-fermion final states in e+ e- collisions," Comput. Phys. Commun. 130 (2000) 260

4. S. Jadach, W. Placzek and B. Ward, Phys. Lett. B390 (1997) 298

5. N. Arkani-Hamed, S. Dimopoulos and G.Dvali, Phys. Lett. B429 (1998) 263

6. Phys. Rev. Lett., 82 (1999) 4765

7. W. Buchmüller, R. Rückl and D. Wyler, Phys. Lett B191 (1987) 442 